\section{Bridges will replace walls...}

According to the 2008 World Bank Development Report, it is now recognised that Agricultural Research for Development (ARD) can clearly contribute to agricultural growth, and returns on investments in agricultural research are considered high. Too often, and particularly in developing countries, ARD is disconnected from the rural world. It is clear that research outcomes cannot have the expected impact if the link with farmers is weak. In order to address this critical situation, effective dissemination mechanisms are needed to allow farmers to use the technology and innovation generated. In line with rapid changes in agriculture and the market environment, new approaches must replace the traditional extension based on a linear, top-down approach, linking research to farmers mainly through public extension services.

The lessons learned have led to a new research and dissemination paradigm, involving rural and agricultural innovation systems which bring together the public and private sectors with farmers, civil society organisations and the scientific community with a view to creating effective linkages between them.

Fruits and vegetables are very well placed for such an approach. To draw major benefits from their very high potential in terms of income generation and health, a multi-stakeholder and multi-sectorial approach is required.
To promote consumption of fruits and vegetables for better health, it is necessary to improve their availability to the consumer, i.e., production, transport, trade, etc. Public-private partnerships are needed in the three main sectors which are mobilised: horticulture, health and education. Innovation and research are key drivers for supporting such an initiative.

Rural and agricultural innovation systems must be set up at local, national and regional levels to bring together all stakeholders from the civil society, private sector, research and extension. These innovation systems can be multiform: from a thematic technology platform associating the private sector and research to operational bodies at local, national and regional levels.

Yesterday, the focus was on multidisciplinary research; today, we must add a multi-stakeholder approach and, tomorrow, the best ways will include the multi-sectorial dimension with a strong interaction between crop scientists, health specialists, nutritionists and people from education.

Bridges will replace walls...

Dr. Jacky Ganry

Chairman of the Section on Tropical and Subtropical Fruits

ISHS 


\section{Éditorial}

\section{Les ponts remplaceront les murs...}

Selon le rapport 2008 de la Banque mondiale sur le développement, il est maintenant reconnu que la recherche agronomique pour le développement (RAD) peut clairement contribuer à la croissance agricole. Les retours sur investissement dans la recherche agronomique sont considérés comme importants. Mais trop souvent, et en particulier dans les pays en voie de développement, l'RAD est trop déconnectée du monde rural. Il est clair que les résultats de la recherche ne peuvent pas avoir l'incidence escomptée si les liens avec les producteurs sont faibles. Pour contrer une telle situation, des mécanismes efficaces de diffusion sont nécessaires afin de permettre aux producteurs de bénéficier des technologies et innovations produites. Considérant les changements rapides de l'environnement de l'agriculture et des marchés, de nouvelles approches doivent remplacer la vulgarisation traditionnelle basée sur une approche linéaire et descendante, liant la recherche aux producteurs principalement par les services publics de vulgarisation.

L'expérience acquise a mené à un nouveau paradigme de recherche et de diffusion des innovations, mettant en ouvre des systèmes d'innovation rurale et agricole qui rassemblent les secteurs publics et privés avec les producteurs, les organismes de la société civile et la communauté scientifique en vue de créer les liens efficaces entre eux.

Les fruits et légumes sont très bien placés pour développer une telle approche. Afin de tirer le meilleur profit de leur potentiel très élevé en termes de revenus et de santé, une approche multi-partenariale et multisectorielle s'impose.

Pour favoriser la consommation des fruits et légumes propice à une meilleure santé, il est nécessaire d'améliorer leur disponibilité auprès du consommateur, c'està-dire leur production, transport, commerce, etc. Des partenariats public-privé sont nécessaires dans les trois secteurs principaux qui sont mobilisés : horticulture, santé et éducation. L'innovation et la recherche sont les moteurs principaux pour soutenir de telles actions.

Des systèmes d'innovation rurale et agricole doivent être mis en place aux niveaux local, national et régional pour rassembler tous les acteurs de la société civile, du secteur privé, de la recherche et de la vulgarisation. Ces systèmes d'innovation peuvent être multiformes : de la plate-forme thématique de technologie associant le secteur privé et la recherche aux instances opérationnelles aux niveaux local, national et régional.

Hier, l'accent était mis sur la recherche multidisciplinaire ; aujourd'hui, il faut y ajouter une dimension multi-partenariale et, demain, la dimension multisectorielle sera indispensable avec une interaction forte entre les agronomes, les spécialistes de la santé, les nutritionnistes et les personnes en charge de l'éducation.

Les ponts remplaceront les murs...

Dr. Jacky Ganry

Président de la section Fruits tropicaux et subtropicaux 